\title{
The Effects of Early Exposure to Thimerosal on Impairments of Social and Stereotyped Behaviors and the Number of Purkinje Cells of Cerebellum in Rats
}

\author{
Zahra Namvarpour ${ }^{1}$, Abdollah Amini2 ${ }^{*}$, Mohammad Nasehi ${ }^{*}{ }^{*}$ Mohammad-Reza Zarrindast $^{3}$ \\ ${ }^{1}$ Institute for Cognitive Science Studies (ICSS), Tehran, Iran \\ ${ }^{2}$ Department of Biology and Anatomical Sciences, Faculty of Medicine, Shahid Beheshti University of Medical Sciences, Tehran, \\ Iran \\ ${ }^{3}$ Cognitive and Neuroscience Research Center (CNRC), Tehran Medical Sciences Branch, Islamic Azad University, Tehran, Iran
}

Corresponding Author: 1. Abdollah Amini; Assistant professor, Department of Biology and Anatomical Sciences, Faculty of Medicine, Shahid Beheshti University of Medical Sciences, Tehran, Iran. Tel: +98 9126898452, Email: d.amini2008@yahoo.com 2. Mohammad Nasehi: Assistant Professor, Cognitive and Neuroscience Research Center (CNRC), Tehran Medical Sciences Branch, Islamic Azad, Tehran, Iran. Email: nasehi@iricss.org

Received April 26, 2018; Accepted July 21, 2018; Online Published September 30, 2018

\begin{abstract}
Introduction: Existing evidence on the impact of thimerosal (THIM), acting to preserve pharmaceutical products (a preservative), on fetal neurodevelopment is very controversial. Here, we investigated the neonatal administration of THIM on behaviors including (1) locomotor activity, (2) social behaviors, and (3) stereotyped behaviors in rats. Since the development of cerebellum continues for some time after birth and it is very imperative in movement, balance, and sensory integration, the number of cerebellum Purkinje cells also were counted.

Materials and Methods: The experiments were directed on 40 young male and female Wistar rats, which were randomly distributed into 4 groups including experimental (male \& female) and control (male \& female) groups. Each rat in the test groups were intramuscularly received $240 \mathrm{\mu g} \mathrm{Hg} /$ $\mathrm{kg}$ THIM on postnatal days $(7,9,11,15)$, while the control contributors received saline in the same pattern. After drug interventions on the fourth postnatal week, rats were evaluated by open field test, and in eighth postnatal week, the test of three-chamber paradigm was performed on animals. At the end of the behavioral tests, histological studies were done.

Results: Rats which were exposed to the THIM displayed impairments of locomotor activity and their social interactions were reduced. While the duration of freezing/grooming as stereotyped behaviors were increased significantly. The results of histological studies also showed a noteworthy decrease in the number of Purkinje cells in both sexes.

Conclusions: These data prove that early postnatal exposure of children to THIM causes permanent neurobehavioral and histological impairments and if similar alterations occur in children exposed to THIM/mercurial agents, neurodevelopmental disorders may happen.

Keywords: Thimerosal, Behavioral Impairments, Cerebellum

Citation: Namvarpour Z, Amini A, Nasehi M, Zarrindast MR. The effects of early exposure to thimerosal on impairments of social and stereotyped behaviors and the number of Purkinje cells of cerebellum in rats. J Appl Biotechnol Rep. 2018;5(3):105-111. doi:10.29252/jabr.05.03.04.
\end{abstract}

\section{Introduction}

Thimerosal (sodium ethylmercurithiosalicylate or THIM containing $49.55 \%$ mercury by weight) is found in some pharmaceutical products and has been applied for many decades as a preservative in a number of drug and biological products, including many infant vaccines. ${ }^{1}$ This substance is first processed to ethylmercury, and after that it changes to inorganic mercury mixtures in the body, which accumulates in the central nerve system and other body structures..$^{2-4}$ Studies have shown that all types of mercury are highly toxic and have many destructive effects on the structure of the brain. Mercury depletes the glutathione (GSH) and destroys other antioxidants, antioxidant defense, enzymes and receptor function, as well as it makes widespread alterations in the three-dimensional protein structure and at times can change them to the internal antigens to destroy the immune system. ${ }^{5}$ A significant amount of mercury was found in the infant's blood that exposed to the THIM-containing vaccines. Studies on monkey infants indicate that, after receiving THIMcontaining vaccines, mercury accumulation in the brain is far more than blood and may persist for months or even years. ${ }^{6}$ The amount of mercury accumulated in the brain of infants is so high that can produce neurotoxic effects and may destroy nerve cells. This early exposure to mercury products, especially THIM, is recognized as one of the contributing factors in the pathogenesis of many kinds of neurodevelopmental disorders, like autistic spectrum disorders (ASDs) or autism. ${ }^{7-10}$

ASD or autism is defined as a multifaceted neurodevelopmental disorder. It was recognized by an impaired ability to engage in social relationships, without

Copyright $\odot 2018$ The Author(s). This is an open-access article distributed under the terms of the Creative Commons Attribution License (http:// creativecommons.org/licenses/by/4.0), which permits unrestricted use, distribution, and reproduction in any medium, provided the original work is properly cited. 
meaningful gestures or other nonverbal and verbal communication skills, and repetitive or stereotyped behaviors. ${ }^{11}$ Even though ASD is described behaviorally by the Association of American Psychiatric, some problems such as physical or health problems are also seen in ASD. In past, autism as a rare disorder was estimated with a prevalence of 2 to 4 in 10000 , but in recent years, this prevalence has shown a significant increase. According to a study in 2016, the estimated prevalence of ASD was 14.6/1000 (1/68) children aged approximately 8 years in the United States. ${ }^{12}$ In this regard, today, ASD is known as a serious problem of public health. Therefore, if mercury has any contribution in the etiology of ASD, the symptoms of autism should be parallel to the symptoms of mercury poisoning. ${ }^{13,14}$ In 2000 , Bernard and colleagues reported similarities between the symptoms of autism and exposure to mercury and described the common brain changes between these two disorders. ${ }^{15}$ Based on these studies, many clinical signs of mercury poisoning, such as social impairments, depression, anxiety, limited eye contact, loss of speech abilities, multiple sensory and motor problems, significant cognitive impairment, and repetitive stereotypical behaviors are similarly seen in the patient with ASD. These similarities isalso observed in biochemistry, neurotransmitters, and neuroanatomical traits. ${ }^{14}$ Different studies in laboratory conditions have tested this hypothesis. Olczak and colleagues, along with several relevant studies, examined different neurobiological effects of THIM in rats. ${ }^{16}$ In one of early studies, the effects of THIM on opioid receptors (morphine, MOR) were measured in the caudate nuclei, periaqueductal gray maters, putamen nuclei, and hippocampus formation of the mice. In their experiment, the mice received 4 different doses of the drug $(12,240,1440$, and $3000 \mu \mathrm{g} \mathrm{Hg} / \mathrm{kg})$ in the second week after birth. The Neonatal administration of THIM caused a dose-dependent surge of MOR densities in the caudate putamen and periaqueductal gray maters but MOR density decreased in the hippocampus (dentate gyrus part), where consequences were complemented with loss of synaptic vesicle marker (synaptophysin) and deteriorating neurons. These findings shows that THIM exposure during early life after birth may cause abnormal neuropathological alterations and an interrupt in the brain development. ${ }^{15}$ In another study, they assessed the early administration effects of THIM (4 i.m. injections, 12 or $240 \mu \mathrm{g}$ THIM-Hg/ $\mathrm{kg}$ on the postnatal days $7,9,11$, and 15) on the brain in Wistar rats. They were detected several neuropathological variations in these adult rats. These variations and abnormal changes included (1) the degeneration of the nerve cells in the prefrontal, temporal cortex, hippocampus formation, and the cerebellum, (2) Blood vessel abnormalities in the temporal cortex, (3) reduced reaction of synaptophysin in the hippocampus formation, (4) Concomitant astroglial atrophy and astrogliosis in the hippocampus and cerebellum cortex, and (5) apoptosis in Bergmann astroglia (positive caspase-3 response). These results showed THIM, at doses comparable to those used in infant vaccines or higher, have a neurotoxic effects during developing rat brain, this suggests the role of this drug in abnormal brain development(neurodevelopmental disorders). ${ }^{16}$ In the last experiment, Olczak et al investigated the impact of neonatal treatment with THIM (with doses of 12, 240,1440 , and $3000 \mu \mathrm{g} \mathrm{Hg} / \mathrm{kg}$ ) on behaviors usually changed in autism like anxiety, motor activity, social communication skills, and learning, in Wistar rats in both sexes. ${ }^{17}$ Adult rats, which were received THIM during the early life after birth, revealed locomotors deficiencies and increased in anxiety to neophobic ratio in the open field test.

The administration of THIM with the highest dose to the both of rats (female and male rats) causes a significant reduction in social communication skills, while the incidence of asocial/antisocial communications was distended in male rats, but decreased in female rats. These finding showed that early postnatal administration of THIM to the infants causes permanent neurobehavioral damages and neurochemical alterations in the central nervous system (CNS), depending on gender and dose level. They concluded that because of the creation of similar pattern changes in THIM/mercurial-exposed children, they could contribute to neurodevelopmental disorders like ASD. ${ }^{17,18}$ At the same time, there are many pieces of evidence of degeneration and degradation of granular and Purkinje cells in the cortex of cerebellum because of exposure to mercury. These damages include degenerative changes, mercury accumulation, and significant cell death. ${ }^{19}$ The most stable neurological impairments found in patients with autism include loss and decreased density of cerebellar Purkinje cells, and cerebellar atrophy. On the other hand, limited studies have been conducted on the toxic effects of mercury compounds, especially THIM, on the tissue structure and number of Purkinje cells, especially in both sexes together. In this regard, we tried to investigate THIM neurotoxic effects in a series of behavioral and cerebellar tissue abnormalities in both sexes. These changes were evaluated following administration of THIM with a higher dose than what is given in the pediatric vaccine.

\section{Materials and Methods}

Experimental Procedures

The experiments were carried out on 40 adult male and female Wistar rats, in groups of 10, which were postnatally treated with intramuscular injection of different doses of THIM (The production and research complex of Pasteur Institute of Tehran, Iran). The newborn animals from these rats were firstly accommodated in a room under standard temperature $=22 \pm 1{ }^{\circ} \mathrm{C}$, at $60 \%$ percent relative humidity, and $12 \mathrm{~h}$ light/dark cycle (lights on at 7:00 AM). Throughout the experiments, all of the animals were freely provided food and water at libitum. In this study, the animals were randomly separated into 4 groups: Rats of each group were placed in special cages of the same size $(20 \times 20 \times 40 \mathrm{~cm})$. Two first groups were considered as the control groups, and 2 next groups were considered as experimental groups and treated as follows:

1. Control group of male Wistar rats which received saline injection

2. Control group of female Wistar rats which received saline injection

3. Experimental group of male Wistar rats exposed to 


\section{THIM}

4. Experimental group of female Wistar rats exposed to THIM.

In experimental groups, THIM (Sigma, Germany) was administered on the $7,9,11$, and 15 postnatal days in one dose 240 microgram per kilogram body weight $(\mu \mathrm{g} / \mathrm{Hg}$ per $\mathrm{kg}$ ) in a final volume of $50 \mu \mathrm{L}$, intramuscularly, according to imitation of childhood vaccination timing pattern, devised by Hornig et al. ${ }^{19,20}$ The cumulative volume of mercury injected in the first 6 months after birth is $187.5 \mu \mathrm{g}$, and the effects of THIM at very low doses (similar to doses used for human infants) in animals have been controversial. ${ }^{19,20}$ Additionally, rats are less sensitive to toxic exposures than humans. Thus, in the current study, the dose of THIM was selected relatively higher to compare subjectively and quantifiably the effects of pharmacologically higher dose of the drug. Control groups received vehicle (saline). All injections were directed like the former method. After drug interventions, 4 weeks after giving birth, all rats were examined by open field test for assessing the locomotor activity, and in the eighth week after birth, social interaction test was accompanied to measure social communication skills, which is known as Crawley's sociability and a favorite for social novelty protocol. After behavioral tests, animals were anesthetized by chloroform. In all groups, body weights of newborn rats were measured with a digital scale before necropsy. After killing the animal, using a scalpel blade or scissors, we made a longitudinal incision along the sagittal suture and a transverse slot along the coronal suture in the base of their skull. After removing the skull wall and a transverse incision in the medulla oblongata, the skull was separated from the spinal cord, and the cerebellum as a whole was removed from the cranial cavity with precision. The cerebellar weight was measured in all neonates with a digital scale. Then, the cerebellum was fixed in $10 \%$ formalin, and tissue preparations were made using routine histological procedures.

\section{Estimation and Count the Purkinje Cell in Cerebellar}

For evaluation and counting the Purkinje cells, ten sections per each block were selected by a random sampling manner for numbering cerebellar Purkinje cells. The lateral, and medial nuclei were measured collectively using a projecting microscope. A computer attached to a microscope (Nikon E200, Nikon, Japan) and immersion lens with numerical aperture $(\times 40$ oil $)$ were applied for estimation of total number of the neurons in cerebellar through the optical dissector method. The fields of microscope were selected by moving the microscope stage in an equal interval using a stage micrometer and systematic uniform random sampling. The $\mathrm{Z}$-axis movement of the microscope stage was calculated by a microcator mounted on the stage. Briefly, an unbiased counting frame with inclusion (right and upper) and exclusion (left and lower) borders was superimposed on the images of the sections viewed on the monitor. To analyze the suitable guard zone and the height of the dissector (h), Z-axis distribution of the nuclei was plotted. The counted neurons were scored and grouped in 10 bins from the percentiles 0-100 through the cerebellar tissue section from the upper
$(0 \%)$ to the lower surface (100\%). The upper and lower $10 \%$ of the histogram were measured as the guard zones, and the counting box was located at the remaining $80 \%(\mathrm{~h})$. For getting a histogram output, the under sampling was balanced out and corrected. The cells were only calculated if they met the following standards: Any nucleus coming into the maximal focus within the sampling box was selected if it was located entirely or partly inside the counting frame and did not touch the exclusion line. The total number of Purkinje cell in cerebellum was estimated by multiplying the numerical density $(\mathrm{Nv})$ by the total volume of deep cerebellar nuclei. $\Sigma$ Q-t Nv (Purkinje cell) =×a BA $\Sigma$ P $\times(-) \times$ h where " $\Sigma$ Q-" was the number of the nuclei coming into focus during scanning the dissector height.

On the average, 100-130 Purkinje cell were counted per animal. " $\Sigma \mathrm{P}$ " was the total counting of the unbiased counting frame in all fields. Also, " $h$ " was the height of the dissector, "a/f" was the frame area (here $37 \mu \mathrm{m} \times 37 \mu \mathrm{m})$ ), " $\mathrm{t}$ " was the real section thickness calculated in every sampled field using the microcator ( $22 \mu \mathrm{m}$ on the average), and BA was the block advance of the microtome which was set at $27 \mu \mathrm{m}$. The total number of Purkinje cells was assessed by multiplying the numerical density $(\mathrm{Nv})$ by the $\mathrm{V}$ (deep cerebellar nuclei). ${ }^{21}$

\section{Behavioral Tests}

The Open Field Test

After fourth postnatal week, all rats were studied by open field test. All tests was conducted in a room with poor lighting in silence and without any previous habituation. Rats were at first kept in the test room for 15 minutes preceding the trial for acclimatization. The Test chamber was white, square, with sizes of $72 \times 72 \mathrm{~cm}$ and $36 \mathrm{~cm}$ height walls. The floor of room was shared into 16 squares of $18 \times 18 \mathrm{~cm}$. Each test lasted for 5 minutes. Line passage Frequency with which the animal go across one of the lines with all 4 paws was recorded by the camera and manually detected and calculated. The test was done according to the Crawly et al protocols. ${ }^{22}$

\section{Three-Chamber Paradigm Test}

In the eighth week after birth, animals were prepared for threechamber paradigm test. This test used for assessment of social communication skills which is known as Crawley's sociability and preference for social novelty protocol. The device used for this test comprises of a rectangular three-chamber box. The dimension of each chamber is $38 \times 90 \mathrm{~cm}$ with separating walls. There are two equal wire glass-like sections with detachable ends that are large enough to hold a single rat and placed vertically in the device. The role of these sections is keeping the familiar/unfamiliar rat. Experiments were done in a 650 lux room lighting, and factors such as social novelty/ preference, social affiliation, self-grooming, and freezing were checked and recorded by the human viewer. There were 3 main phases for three-chamber paradigm test:

\section{Habituation phase}

During this phase, right and left portions of the box were separated. Empty wire restraints were placed in the center of both chambers. The rat was positioned at the center of the 
middle section for 5 minutes' accommodation.

\section{Social Affiliation and Sociability Phase (Session I)}

For assessing of social affiliation in the tested animals, one control rat (Stranger 1) was located inside a wire cup in one of the lateral spaces. Then, the walls between each section were removed to allow the rat to discover each of the three sections. Phase's length was 10 minutes and duration of direct communications between the subject animal and the "Stranger 1" rat (containment lodging or not lodging), independently, and the time of other behaviors by the experimental rat, containing self-grooming, and un-movements for more than 5 seconds (freezing) were monitored and recorded.

\section{Social Novelty/Preference and Social Memory Phase (Session II)}

In the final session of excrement, social memory and novelty was tested. For this, a second control animal (stranger 2) was located into the other wire cup in the opposed side (that had been empty during the session I) for characteristic the behaviors between the test rat in the presence of stranger 1 compared with Stranger 2 and the same parameters described in session I were observed and recorded. This session's time was also 10 minutes.

\section{Statistical Analyses}

In this experiment, one-way ANOVA, Tukey, and KruskalWallis analyses were applied in order to evaluate locomotor activities, stereotype behaviors and the data obtained from tissue tests. Student's $t$ test was also used to evaluate social behaviors in three-chamber paradigm test. All statistical tests were directed by SPSS version 21 and significantly were considered at $P<0.05$.

\section{Ethical Considerations}

The protocol used in this experiment was confirmed by the institutional Medical Ethics Commission of Shahid Beheshti University of Medical Sciences (No. IR. SBMU. MSP. REC.1395.167).

\section{Results}

\section{Locomotor Activates}

The results in Table 1 indicate that high-dose THIM (240 $\mu \mathrm{g} / \mathrm{kg}$ ) in both males and females decreased motor activity $(P<0.001)$. However, there is no significant alteration between male and female animals in motor activity.

\section{Social Behaviors Following Treatment With High Dose of THIM \\ Session I}

As is illustrated in Figure 1A, independent $t$ test results show that no significant difference is between the means of the stranger 1 and empty chamber in male and female rats treated with THIM $(240 \mu \mathrm{g} / \mathrm{kg})$ (male rats $P=0.07$, Female rats $P=0.143$ ). These results indicate a less natural tendency for these animals to establish social affiliation and sociability. In the control groups that received normal saline, there is a
Table 1. The Effect of THIM $(240 \mu \mathrm{g} / \mathrm{Hg} \mathrm{kg})$ on Locomotor Activity in Open Field Test

\begin{tabular}{lc}
\hline Experimental Groups & $\begin{array}{c}\text { Frequency of Passage of } \\
\text { Lines in } \mathbf{5} \text { min }\end{array}$ \\
\hline Control (male Wistar rats) & $42.526 \pm 4.25$ \\
Control (female Wistar rats) & $40.25 \pm 3.20$ \\
Thimerosal $(3000 \mu \mathrm{g} / \mathrm{kg})$ male Wistar rats & $7.6 \pm 1.4^{* * *}$ \\
Thimerosal $(3000 \mu \mathrm{g} / \mathrm{kg})$ female Wistar rats & $11.08 \pm 3.17^{\sharp \# \#}$ \\
\hline
\end{tabular}

Values are mean $\pm \operatorname{SEM}(n=10)$. As shown in the table, there are a significant difference between THIM male rats as compared to control male rat, $P<0.001^{* * *}$, as well as, a significant difference between THIM female rats in compared to female control group $P<0.001^{\sharp \#}$ were observed in this study.

significant change between the stranger 1 and empty chamber relation $(P<0.001)$.

\section{Session II}

In the second session, the subject rats were allowed to choose to communicate with the animal that they have already interacted with (stranger 1) or the rat that has just entered the empty chamber (stranger 2). Accordingly, the animal's tendency to communicate with the newer rat represents a healthy social memory and the preference for novel experiences. The results of the test in Figure $1 \mathrm{~B}$ showed that male and female rats receiving high doses of THIM $240(\mu \mathrm{g} /$ $\mathrm{kg}$ ) were reluctant to interact with the new animal, and there was no significant difference between the association with stranger 1 and stranger 2 (Male rats $=0.45$, female rats $=0.47$ ). A significant change between the stranger 1 and stranger 2 in control groups, in second session, revealed intact social memory and novelty $(P<0.001)$.

Stereotype Behaviors After Treatment With THIM in High Dose

The results of one-way ANOVA test in Figures 2A and B show

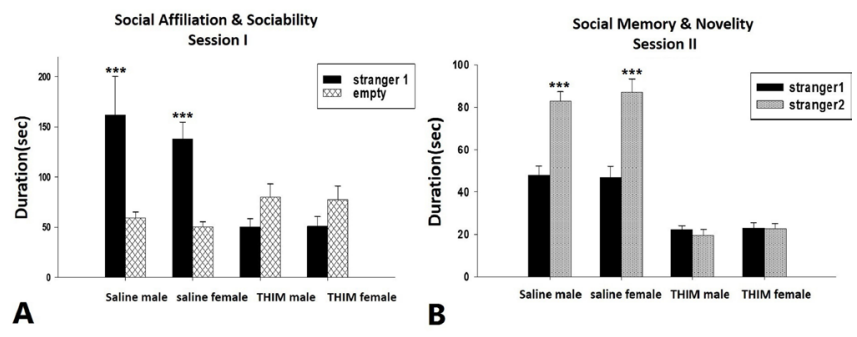

Figure 1. Three-Chamber Paradigm Test. (A) Session I of three-chamber test, social affiliation and sociability phase. Time length in the chamber with stranger compared to the opposite chamber in 8-week old control and THIM treated rats. Animals which treated with THIM, unlike saline group, failed to show a preference of social nearness by spending nonsignificant time in both chambers: (B) Session II of three-chamber test. Stranger 2 was added under the empty cup for a 10-min social memory \& novelty test. Mean duration of time $( \pm$ SEM) in the chamber with the unfamiliar animal from sociability phase ("stranger 1 ") and the opposite chamber with a new unfamiliar rat ("stranger 2"). Unlike saline group, animals which treated with THIM, were unsuccessful to demonstrate a preference for social novelty and failed in social memory by spending approximately same time in both chambers. 


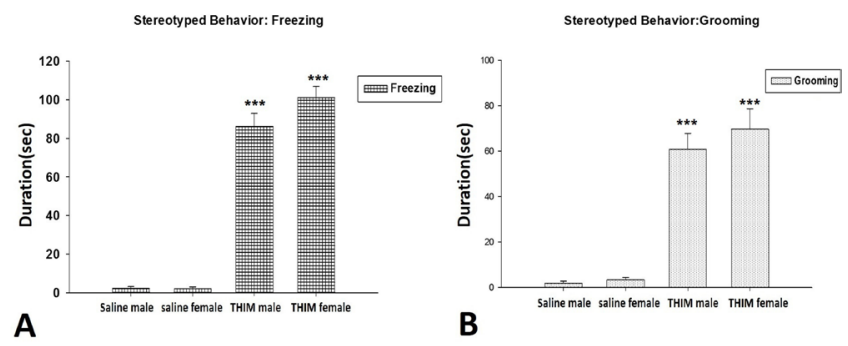

Figure 2. Effect of THIM Administration on Stereotyped Behaviors (Freezing \& Grooming), in the Social Interaction Three-Chamber Test. (A) duration of freezing, in rats treated with high doses of THIM, which represent statistically significant increase in comparison to groups. (B) duration of Grooming, in rats treated with THIM, which represent statistically significant increase in comparison to saline group. Data presented as mean \pm SEM. Sign $\left(^{* * *}\right)$ compared with control groups $(P<0.001)$.

that the stereotyped and repetitive behaviors (freezing and grooming) in male and female rats treated with high doses of THIM were significantly increased $(P<0.001)$. However, the difference between sexes is not meaningful.

Determine the Number of Purkinje Cells in the Cerebellum Figures 3 and 4 show a part of the cerebellum in the control (Cont.M and Cont.F) and THIM exposed groups (EXP.M and EXP.F). As it can be seen in the picture, the 3 layers of the cerebellum cortex, including the molecular layer (M), which is observed in low color staining. The Purkinje cells (P) layer which are characterized by a clear nucleus, and a large and uniform cytoplasm is regularly arranged in a row. As shown in Figures 3 and 4, the trunk of these cells is large and pearshaped and between two granular and molecular layers. The Granular layer (G) is composed of small and compact droplets of congested cells that can be seen in the image.

Figures 3 and 4 show a fraction of the cerebellum in the experimental groups of male(EXP.M) and female mice(EXP.F), respectively, treated with THIM with a similar magnification as the control groups (Figure 3 Cont.M and Cont.F). As is displayed in Figures 3 and 4, it is detectable in the cerebellum cortex of the 3 layers of P (Purkinje layer), M (Molecular layer), and $\mathrm{G}$ (Granular layer). The difference in experimental group (Figures 3 and 4) was the decrease in the number of Purkinje cells compared to the control group. The mean of Purkinje cells in the male experimental groups (EXP.M) was $5.18 \pm 1.05$ and in the male control group(Cont.M) was $6.06 \pm 1.52$. Statistical analysis showed that this difference was significant $(P<0.01)$. The mean of Purkinje cells in the female experimental group(EXP.F) was $5.24 \pm 0.73$ and in the female control group(Cont.f) was $6.31 \pm 0.079$ which is statistically significant $(P<0.01)$. This difference was not significant in the 2 experimental groups $(P=0.11)$.

\section{Discussion}

A limited number of studies about the various types of mercury effects on the developing of nervous system discusses the effect of THIM on motor activates and social and stereotyped or repetitive behaviors. However, until now, there is no reports to show the high dose effects of THIM



Figure 3. Photomicrograph of Rat Cerebellum in the Studied Groups (10x). Cont. $\mathrm{M}=$ Control Male and Cont. $\mathrm{F}=$ Control Female and EXP. $\mathrm{M}=$ Experimental Male and EXP. F=Experimental Female groups by staining $\mathrm{H} \& \mathrm{E}$ and magnifying 10x. Molecular layer (M), Purkonje layer $(\mathrm{P})$, Granular layer $(\mathrm{G})$, and White material (W).

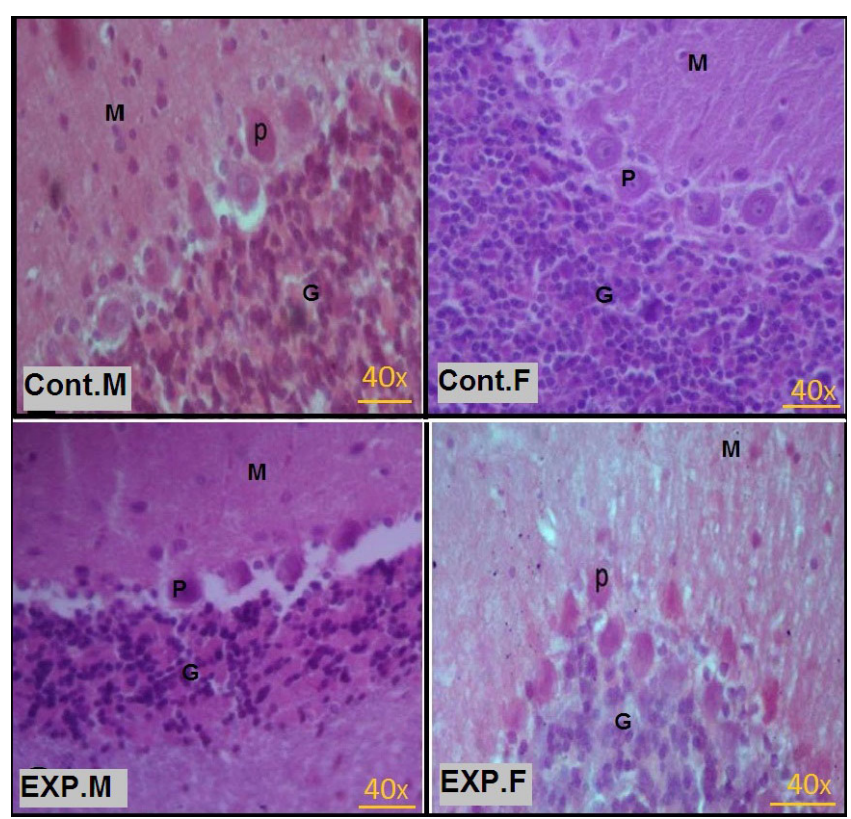

Figure 4. Photomicrograph of Rat Cerebellum in the Studied Groups (40x). Cont. $\mathrm{M}=$ Control Male and Cont. $\mathrm{F}=$ Control Female and EXP. $\mathrm{M}=$ Experimental Male and EXP. $\mathrm{F}=$ Experimental Female groups by staining $\mathrm{H}$ \& $\mathrm{E}$ and magnifying 40x. Molecular layer (M), Purkonje layer $(\mathrm{P})$, Granular layer $(\mathrm{G})$, and White material (W).

on the behavioral and histological characteristics of male and female rats. The present study shows that motor activity in rats treated with THIM in high dose has significantly decreased in both sexes. The findings are similar to other studies that have revealed a decrease in motor activity even at the lowest dose of THIM. ${ }^{17,19,23}$ Among them, the present finding is in accordance with the report of Olzak et al. They 
showed that motor dysfunction and neophobic/anxiety were observed in all rats treated with different doses of THIM. ${ }^{17}$ Our findings are also consistent with Hornig et al results in which the permanent decrease of motor activity was observed in animals postnatal exposed to THIM. ${ }^{19}$ The results of Sulkowski and colleagues' research on prenatal exposure to the THIM in male and female rats also confirmed the damage to the cerebellum due to oxidative stress and reduced motor activity in both sexes. ${ }^{23}$ Then, the effects of different types of mercury on locomotor activities have been likely pronen in several studies. ${ }^{24}$ In another study by Olczak et al, the damage to the Purkinje cells of the cerebellum has been reported in the mice treated with THIM. ${ }^{16}$ Another study reported the induction of metallothionein (MT) mRNA after exposuring the mouse brain to the low doses of THIM. ${ }^{25}$

Regarding the results of these studies and the present study, it seems that the cerebellum has a high sensitivity to the THIM and the disturbance in motor activity may be due to damaging the cerebellum after exposure to THIM. High dose THIM- treated rats also revealed a decrease in levels of social affiliation and sociability throughout the session I of threechamber test (Crawley's sociability and preference). During this session, decreased rate of sociability and social closeness was seen, and here rats spend approximately more time around the empty cup, than social interaction with another rat, which shows an abnormal altered sociability, affiliation, and social motivations. In the second session, reduced rate of novelty and social memory was mentioned. Rats were failed to show a preference for social novelty and social memory test by spending the same time in both chambers. These dimorphic changes in social interactions are consistent with Olczak et al results, suggesting that early exposure of neonatal animals to THIM causes a social interaction deficit in adult animals in dose- dependent manners. ${ }^{17}$ The results of the present study, along with other studies, indicate the presence of neurological degeneration due to exposure to the THIM. ${ }^{26}$ It seems that high doses of THIM causes a variety of behavioral disorders similar to neurodevelopmental disorders such as autism through nerve degeneration.

As far as our knowledge, it is the first time documenting that neonatal high dose THIM treatment induces a permanent increase in repetitive and stereotyped behaviors in both sexes. Our clinical observations, of course, indicate that there are severe aggressive behaviors in rats receiving high dosage of THIM, suggesting a precise examination of this behavioral disorder is necessary in the future. However, the stereotyped and repeatable behaviors might be due to impaired glutaminergic system in the brain. ${ }^{27,28}$ Also, there are many works which address abnormalities associated with glutamate remarkably similar in poisoning by mercury and ASD. ${ }^{13,14,29,30}$ Therefore, it is likely that postnatal exposure of rats to THIM can induce lasting and abnormal stereotypic and repetitive activities which could partially associated to the abnormal function of the glutamatergic system.

\section{Conclusions}

The results of this study revealed that the Purkinje cells density in the cerebellum of rats exposed to high dosage of THIM was significantly lower than control cerebellum. This decrease in number could be due to the decreased activity of the nucleus, decreased cell activity, as well as increased cell death and apoptosis. About the action mechanism of THIM, it is believed that mercury, with its coherent effect, exerts its effect through the local increase of free radicals.

In 2007, Laurente et al, in a study on the effects of THIM on the brain and the cerebellum in hamsters, showed that THIM could reduce the number of cells, demyelination of axonal neoplasms, Purkinje and granular cells necrosis, and glycemia in all layers of the cerebellum. ${ }^{18}$ These findings are supported by the obtained results of this study.

On the whole, the current study offers extra experimental evidence that exposure to THIM leads to neurotoxic changes in the developing brain, providing stronger support to remove this needless component from many vaccines and other products of medical.

\section{Authors' Contributions}

$\mathrm{ZN}$ and $\mathrm{AA}$ had substantial contribution to the conception, research, data acquisition, and analysis/interpretation. $\mathrm{AA}$ and $\mathrm{MN}$ collaborated in the preparation/revision of the submitted manuscript. MN participated in reaching the approval for the publishable manuscript version. MRZ ensured a proper explanation to possible questions that could be raised regarding accuracy and scientific integrity of the submitted manuscript.

\section{Conflict of Interest Disclosures}

The authors have no commercial, proprietary, or financial interest in the products or companies described in the current article.

\section{Acknowledgments}

The present article is financially supported by the "Research Department of the School of Medicine, Shahid Beheshti University of Medical Sciences" (grant No. 8070).

\section{References}

1. Geier DA, Kern JK, King PG, Sykes LK, Geier MR. A case-control study evaluating the relationship between thimerosal-containing haemophilus influenzae type $b$ vaccine administration and the risk for a pervasive developmental disorder diagnosis in the United States. Biol Trace Elem Res. 2015;163(1-2):28-38. doi:10.1007/ s12011-014-0169-3.

2. Barregard L, Rekic D, Horvat M, Elmberg L, Lundh T, Zachrisson O. Toxicokinetics of mercury after long-term repeated exposure to thimerosal-containing vaccine. Toxicol Sci. 2011;120(2):499-506. doi:10.1093/toxsci/kfr009.

3. Dorea JG, Marques RC, Isejima C. Neurodevelopment of Amazonian infants: antenatal and postnatal exposure to methyland ethylmercury. J Biomed Biotechnol. 2012;2012:132876. doi:10.1155/2012/132876.

4. Geier DA, King PG, Hooker BS, et al. Thimerosal: clinical, epidemiologic and biochemical studies. Clin Chim Acta. 2015;444:212-220. doi:10.1016/j.cca.2015.02.030.

5. Kidd PM. Autism, an extreme challenge to integrative medicine. Part 2: medical management. Altern Med Rev. 2002;7(6):472-499.

6. Hewitson L, Lopresti BJ, Stott C, Mason NS, Tomko J. Influence of pediatric vaccines on amygdala growth and opioid ligand binding in rhesus macaque infants: A pilot study. Acta Neurobiol Exp (Wars). 2010;70(2):147-164.

7. Gallagher C, Goodman M. Hepatitis B triple series vaccine and developmental disability in US children aged 1-9 years. Toxicol Environ Chem. 2008;90(5):997-1008. doi:10.1080/02772240701806501.

8. Geier DA, Geier MR. A prospective assessment of porphyrins in autistic disorders: a potential marker for heavy metal exposure. 
Neurotox Res. 2006:10(1):57-64 doi:10.1007/BF03033334.

9. Geier DA, Hooker BS, Kern JK, King PG, Sykes LK, Geier MR. A dose-response relationship between organic mercury exposure from thimerosal-containing vaccines and neurodevelopmental disorders. Int J Environ Res Public Health. 2014;11(9):9156-9170. doi:10.3390/ijerph110909156.

10. Geier MR, Geier DA. Neurodevelopmental disorders after thimerosal-containing vaccines: a brief communication. Exp Biol Med (Maywood). 2003;228(6):660-664. doi:10.1177/153537020322800603.

11. Burket RC, Schramm LL. Therapists' attitudes about treating patients with eating disorders. South Med J. 1995;88(8):813-818.

12. Kern JK, Geier DA, Sykes LK, Haley BE, Geier MR. The relationship between mercury and autism: A comprehensive review and discussion. J Trace Elem Med Biol. 2016;37:8-24. doi:10.1016/j. jtemb.2016.06.002.

13. Kern JK, Geier DA, Audhya T, King PG, Sykes LK, Geier MR. Evidence of parallels between mercury intoxication and the brain pathology in autism. Acta Neurobiol Exp (Wars). 2012;72(2):113153.

14. Carneiro MF, Souza JM, Grotto D, Batista BL, de Oliveira Souza VC, Barbosa F Jr. A systematic study of the disposition and metabolism of mercury species in mice after exposure to low levels of thimerosal (ethylmercury). Environ Res. 2014;134:218227.

15. Olczak M, Duszczyk M, Mierzejewski P, Bobrowicz T, Majewska MD. Neonatal administration of thimerosal causes persistent changes in mu opioid receptors in the rat brain. Neurochem Res. 2010;35(11):1840-1847. doi:10.1007/s11064-010-0250-z.

16. Olczak M, Duszczyk M, Mierzejewski P, Wierzba-Bobrowicz T, Majewska MD. Lasting neuropathological changes in rat brain after intermittent neonatal administration of thimerosal. Folia Neuropathol. 2010;48(4):258-269.

17. Olczak M, Duszczyk M, Mierzejewski P, Meyza K, Majewska MD. Persistent behavioral impairments and alterations of brain dopamine system after early postnatal administration of thimerosal in rats. Behav Brain Res. 2011;223(1):107-118. doi:10.1016/j. bbr.2011.04.026.

18. Laurente J, Remuzgo F, Avalos B, et al. Neurotoxic effects of thimerosal at vaccines doses on the encephalon and development in 7 days-old hamsters. An Fac Med Lima. 2007;68(3):222-237.

19. Hornig M, Chian D, Lipkin WI. Neurotoxic effects of postnatal thimerosal are mouse strain dependent. Mol Psychiatry.
2004;9(9):833-845. doi:10.1038/sj.mp.4001529.

20. Namvarpour Z, Nasehi M, Amini A, Zarrindast MR. Protective role of alpha-lipoic acid in impairments of social and stereotyped behaviors induced by early postnatal administration of thimerosal in male rat. Neurotoxicol Teratol. 2018;67:1-9. doi:10.1016/j. ntt.2018.02.002.

21. Noorafshan A, Erfanizadeh M, Karbalay-Doust S. Stereological studies of the effects of sodium benzoate or ascorbic acid on rats cerebellum. Saudi Med J. 2014;35(12):1494-1500.

22. Gerfen CR, Rogawski MA, Sibrey DR, Skolnick P, Wray S. Short Protocols in Neuroscience: Systems and Behavioral Methods. Hoboken: John Wiley; 2006:610.

23. Sulkowski ZL, Chen T, Midha S, Zavacki AM, Sajdel-Sulkowska EM. Maternal thimerosal exposure results in aberrant cerebellar oxidative stress, thyroid hormone metabolism, and motor behavior in rat pups; sex- and strain-dependent effects. Cerebellum. 2012;11(2):575-586. doi:10.1007/s12311-011-0319-5.

24. Gandhi D, Dhull DK. Postnatal Behavioural Effects on the Progeny of Rat after Prenatal Exposure to Methylmercury. Am J Exp Biol. 2014;1(1):31-51.

25. Minami T, Miyata E, Sakamoto Y, Yamazaki H, Ichida S. Induction of metallothionein in mouse cerebellum and cerebrum with lowdose thimerosal injection. Cell Biol Toxicol. 2010;26(2):143-152. doi:10.1007/s10565-009-9124-z.

26. Li B, Zhang Y, Ma D, Shi Z, Ma S. Mercury nano-trap for effective and efficient removal of mercury(II) from aqueous solution. Nat Commun. 2014;5:5537. doi:10.1038/ncomms6537.

27. Lewis $\mathrm{MH}$, Tanimura $\mathrm{Y}$, Lee LW, Bodfish JW. Animal models of restricted repetitive behavior in autism. Behav Brain Res. 2007;176(1):66-74. doi:10.1016/j.bbr.2006.08.023.

28. Afsordeh K, Sadeghi Y, Amini A, et al. Alterations of neuroimmune cell density and pro-inflammatory cytokines in response to thimerosal in prefrontal lobe of male rats. Drug Chem Toxicol. 2018:1-11. doi:10.1080/01480545.2018.1465949.

29. de Oliveira Souza VC, de Marco KC, Laure HJ, Rosa JC, Barbosa $\mathrm{F}$ Jr. A brain proteome profile in rats exposed to methylmercury or thimerosal (ethylmercury). J Toxicol Environ Health A. 2016:79(12):502-512. doi:10.1080/15287394.2016.1182003.

30. Mutkus L, Aschner JL, Syversen T, Shanker G, Sonnewald U, Aschner M. In vitro uptake of glutamate in GLAST- and GLT-1-transfected mutant $\mathrm{CHO}-\mathrm{K} 1$ cells is inhibited by the ethylmercury-containing preservative thimerosal. Biol Trace Elem Res. 2005;105(1-3):7186. doi:10.1385/bter:105:1-3:071. 